\title{
SOME RESULTS ON CERTAIN SUBCLASSES OF ANALYTIC FUNCTIONS INVOLVING GENERALIZED HYPERGEOMETRIC FUNCTIONS AND HADAMARD PRODUCT
}

\author{
KHALIDA INAYAT NOOR \\ Mathematics Department \\ College of Science \\ King Saud University \\ Riyadh 11451, Saudi Arabia \\ (Received April 26, 1990 and in revised form March 22, 1991)
}

\begin{abstract}
By using a certain linear operator defined by a Hadamard product or convolution, several interesting subclasses of analytic functions in the unit disc are introduced and some unifying relationships between them are established. A variety of characterization results involving a certain functional and some general functions of hypergeometric type are investigated for these classes.
\end{abstract}

Key Words and Phrases: Analytic, Hadamard product, Hypergeometric functions univalent, starlike, convex, close-to-convex, Quasi-convex, Linear operator.

1980 Mathematics Subject Classification: 30A32, 30C45, 30A34.

1. INTRODUCTION. Let $A$ denote the class of the function $f$ of the form

$$
f(z)=z+\sum_{n=2}^{\infty} a_{n} z^{n}
$$

which are analytic in the unit $\operatorname{disc} E=\{z:|z|<1\}$. A function $f \varepsilon A$ is said to be in the class $R(\beta)$ if, for $z \varepsilon E$ and $\beta>-1$,

$$
\operatorname{Re} \frac{z f^{\prime}(z)}{f(z)}>-\beta
$$

Also, a function $f \varepsilon A$ is said to belong to the class $V(\beta)$ if, for $z \varepsilon E$ and $\beta>-1$,

$$
\operatorname{Re} \frac{\left(z f^{\prime}(z)\right)^{\prime}}{f^{\prime}(z)}>-\beta \text {. }
$$

We note that

$$
f(z) \varepsilon R(\beta) \leftrightarrow z f^{\prime}(z) \varepsilon V(\beta),
$$

and $\quad v(\beta) \subset R(\beta)$.

The classes $V(\beta)$ and $R(\beta)$ of analytic functions have been defined and studied in [9].

We define the following.

Let $f \varepsilon A$ and let $g \varepsilon R(\beta)$. Then $f \varepsilon T(\alpha, \beta)$ if, for $\alpha>-1$ and $z \varepsilon E, R e \frac{z f^{\prime}(z)}{g(z)}>-\alpha$.

Also, let $f \varepsilon A$. Then $f \varepsilon T^{*}(\alpha, \beta)$ if, for $\alpha>-1, z \varepsilon E$ and $g \varepsilon V(\beta)$, 


$$
\operatorname{Re} \frac{\left(z f^{\prime}(z)\right)^{\prime}}{g^{\prime}}>-\alpha
$$

From (1.3) and (1.4), it is clear that

and

$$
\begin{gathered}
\int \varepsilon T^{*}(\alpha, \beta) \leftrightarrow z f^{\prime} \varepsilon T(\alpha, \beta) \\
T^{*}(\alpha, \beta) \subset T(\alpha, \beta)
\end{gathered}
$$

Let $f_{j}(z)(j-1,2)$ in $A$ be given by

$$
f_{j}(z)=\sum_{n=0}^{\infty} a_{n+1, j} z^{n+1} \quad\left(a_{i j}=1\right)
$$

Then the Hadamard product (or convolution) $f_{1} * f_{2}(z)$ of $f_{1}(z)$ and $f_{2}(z)$ is defined by

$$
f_{1} * f_{2}(z)=\sum_{n=0}^{\infty} a_{n+1,1} a_{n+1,2} z^{n+1}
$$

Let $a_{j}(j=1, \ldots, p)$ and $\beta,(j=1,2, \ldots, q)$ be complex numbers with $\beta_{j} \neq 0,-1,-2, \ldots, j=1, \ldots, q$.

Then the generalized hypergeometric function ${ }_{p} F_{q}$ is defined by

$$
{ }_{p} F_{q}(z)={ }_{p} F_{q}\left(\alpha_{1}, \ldots, \alpha_{p} ; \beta_{1}, \ldots, \beta_{q} ; z\right)=\sum_{n=0}^{\infty} \frac{\left(\alpha_{1}\right)_{n} \ldots \ldots\left(\alpha_{p}\right)_{n}}{\left(\beta_{1}\right)_{n} \ldots \ldots\left(\beta_{q}\right)_{n} n !} z^{n} \quad(p \leq q+1)
$$

where $(\lambda)_{n}$ is the Pochhammer symbol defined by

$$
(\lambda)_{n}= \begin{cases}1 & \text { if } n=0 \\ \lambda(\lambda+1) \ldots . .(\lambda+n-1) & \text { if } \quad n \varepsilon N=\{1,2,3 \ldots\} .\end{cases}
$$

We now define the function $\phi(a, c)$ by

$$
\phi(a, c, z)=\sum_{n=0}^{\infty} \frac{(a)_{n}}{(c)_{n}} z^{n+1}, \quad(c \approx 0,-1,-2, \ldots, z \varepsilon E)
$$

so that $\phi(a, c)$ is an incomplete Beta function with

$$
\phi(a, c, z)=z_{2} F_{1}(1, a ; c, z) .
$$

Corresponding to the function $\phi(a, c)$, Carlson and Shaffer [2] defined a linear operator $L(a, c)$ on $A$ by the convolution

$$
L(a, c) f=\phi(a, c) * f
$$

for $f \varepsilon A$. Clearly, $L(a, c)$ maps $A$ onto itself, and $L(c, a)$ is an inverse of $L(a, c)$ provided that $a \neq 0,-1,-2, \ldots$

Furthermore, $L(a, a)$ is the identity operator, and

$$
R(\beta)=L(1,2) V(\beta) \text {, and } V(\beta)=L(2,1) R(\beta) \text {. }
$$

Also

$$
T(\alpha, \beta)=L(1,2) T^{*}(\alpha, \beta), \text { and } T^{*}(\alpha, \beta)=L(2,1) T(\alpha, \beta),
$$

where $\alpha>-1$ and $\beta>-1$.

We can now define the classes of analytic function with which we shall be dealing.

Definition 1.1. A function $f \varepsilon A$ is said to be in the class $R(a, c ; \beta)$ if $L(a, c) f$ belongs to $R(\beta)$ for $\beta>-1$, and $f \varepsilon V(a, c ; \beta)$ if, and only if, $z f^{\prime} \varepsilon R(a, c ; \beta)$ for $\beta>-1$.

Similarly we have: 
Definition 1.2. A function $f \varepsilon A$ is said to be in the class $T(a, c ; \alpha, \beta)$ if $L(a, c) f \varepsilon T(\alpha, \beta)$ for $\alpha>-1$ and $\beta>-1$. Further $f \varepsilon T^{*}(a, c ; \alpha, \beta)$ if, and only if, $z f^{\prime} \in T(a, c ; \alpha, \beta)$ for $\alpha>-1$.

The following relations can easily be verified.

$$
\begin{aligned}
V(a, c ; \beta) & =L(1,2) R(a, c ; \beta) \\
R(a, c, \beta) & =L(2,1) V(a, c, \beta) \\
V(\beta) & =V(a, a ; \beta)=L(1,2) R(a, a ; \beta)
\end{aligned}
$$

and

$$
R(\beta)=R(a, a ; \beta)=L(2,1) V(a, a ; \beta)
$$

Also

$$
\begin{gathered}
T^{*}(a, c ; \alpha, \beta)=L(1,2) T(a, c ; \alpha, \beta) \\
T(a, c ; \alpha, \beta)=L(2,1) T^{*}(a, c ; \alpha, \beta) \\
T^{*}(\alpha, \beta)=T^{*}(a, a ; \alpha, \beta)=L(1,2) T(a, a ; \alpha, \beta)
\end{gathered}
$$

and

$$
T(\alpha, \beta)=T(a, a ; \alpha, \beta)=L(2,1) T^{*}(a, a ; \alpha, \beta)
$$

We shall now connect these classes with the univalent functions. A single-valued function $\int$ is said to be univalent in a domain $D$ if it never takes on the same value twice. By $S, K, S^{*}, C$ and $C^{*}$ denote the subclasses of $A$ which are respectively univalent, close-to-convex, starlike, convex and quasi-convex in $E$. In [8], Robertson defined the subclasses of $C$ and $S^{*}$ by using the order of the class as follows. A function $\int \varepsilon S$ is called a convex function of order $\beta_{1}, 0 \leq \beta_{1}<1$, if and only if $\operatorname{Re} \frac{\left(z f^{\prime}(z) y\right.}{f^{\prime}(z)}>\beta_{1}, z \varepsilon E$. We denote this class as $C\left(\beta_{1}\right)$. Also a function $f \varepsilon S$ is called starlike function of order $\beta_{1}, 0 \leq \beta_{1}<1$ if and only if $R e \frac{: f^{\prime \prime}(:)}{\left.N_{i}\right)}>\beta_{1}$, $z \varepsilon E$. We call this class $S^{*}\left(\beta_{1}\right)$. Obviously

$$
f \varepsilon C\left(\beta_{1}\right) \leftrightarrow z f^{\prime} \varepsilon S^{*}\left(\beta_{1}\right)
$$

Libera [3] introduced the terminology of order and type together in the class $K\left(\alpha_{1}, \beta_{1}\right)$ of close-to-convex functions. A function $f \varepsilon a$ is said to be close-to-convex of order $\alpha_{1}$ type $\beta_{1}, 0 \leq \alpha_{1}<1$; $0 \leq \beta_{1}<1$, if and only if there exists a function $g \varepsilon S^{*}\left(\beta_{1}\right)$ such that $\operatorname{Re} \frac{\varepsilon^{\prime}(\xi)}{g^{(z)}}>\alpha_{1}, z \varepsilon E$. Further $f \varepsilon C^{*}\left(\alpha_{1}, \beta_{1}\right) \leftrightarrow z f^{\prime} \varepsilon K\left(\alpha_{1}, \beta_{1}\right)$ we refer to [7].

Indeed from the above definitions of the various subclasses of the various subclasses of $A$, we deduce readily the following:

$$
\begin{aligned}
& S^{*}\left(\beta_{1}\right) \subset S^{*} \subset R(\beta) \subset A, \\
& C\left(\beta_{1}\right) \subset C \subset V(\beta) \subset R(\beta) \subset A
\end{aligned}
$$

and

$$
\begin{gathered}
C^{*}\left(\alpha_{1}, \beta_{1}\right) \subset C^{*} \subset T^{*}(\alpha, \beta) \subset T(\alpha, \beta) \subset A, \\
K\left(\alpha_{1}, \beta_{1}\right) \subset K \subset T(\alpha, \beta) \subset A,
\end{gathered}
$$

where

$$
0 \leq \alpha_{1}<1, \quad 0 \leq \beta_{1}<1 \text { and }-1<-\alpha_{1} \leq \alpha ;-1<-\beta_{1} \leq \beta
$$




\section{MAIN RESULTS}

We first state certain results which will be needed in proving our main theorems.

Lemma 2.1. [6] Let $\phi(u, v)$ be the complex function, $\phi: D \rightarrow C, D \subset C \times C(C$-complex plane $)$ and let $u=u_{1}+i u_{2}, v=v_{1}+i v_{2}$. Suppose that the function $\phi$ satisfies the conditions:

(i) $\phi(u, v)$ is continuous in $D$;

(ii) $\quad(1,0) \varepsilon D$ and $R e\{\phi(0,1)\}>0$;

(iii) $\operatorname{Re}\left\{\phi\left(i u_{2}, v_{1}\right)\right\}<0$ for all $\left(i u_{2}, v_{1}\right) \varepsilon D$ and such that $v_{1} \leq\left(1+u_{2}^{2}\right) / 2$.

Let $h(z)=1+c_{1} z+\ldots$ be analytic in $E$, such that $\left(h(z), z h^{\prime}(z)\right) \varepsilon E$ for all $z \varepsilon E$. If $\operatorname{Re}\left\{\phi\left(h(z), z h^{\prime}(z)\right)\right\}>0(z \varepsilon E)$, then $\operatorname{Reh}(z)>0$ for $z \varepsilon E$.

Let $I_{\lambda}(f)$ denote a functional defined by

$$
I_{\lambda}(f)=\frac{\lambda+1}{z^{\lambda}} \int_{0}^{z} t^{\lambda-1} f(t) d t
$$

for $f \varepsilon A$ and for a real number $\lambda>1$. The functional $I_{\lambda}(f)$, when $\lambda \varepsilon N$, was studied by Bernardi [1], and in particular, $I_{1}(f)$ was considered earlier by Libera [4] and Livingston [5]. We note that $I_{2}(f)$ is a particular solution of the ordinary first order differential equation

$$
\operatorname{tg}^{\prime}(t)+\lambda g(t)(++1) f(t)
$$

at the point $t=z$. Also by comparing (1.9) and (2.1), we have $I_{\lambda}(f)=L(\lambda+2, \lambda+1) f$. For our next results we refer to [9].

Theorem 2.1. Let $g \varepsilon R(a, c ; \beta)$ and let, for $\lambda \geq \beta>-1, I_{\lambda}(g)$ be defined by $(2.1)$. The $I_{\lambda}(g)$ is also in the class $R(a, c ; \beta)$.

We shall now prove the following.

Theorem 2.2. Let $f \varepsilon T(a, c ; \alpha, \beta)$ and let, for $\lambda \geq \alpha, \beta>-1, I_{\lambda}(f)$ be defined by (2.1). Then $I_{\lambda}(f) \in T(a, c ; \alpha, \beta)$.

Proof: Since $f \varepsilon T(a, c ; \alpha, \beta)$, there exists $g \varepsilon R(a, c, \beta)$ such that

$$
\operatorname{Re}\left\{\frac{z[L(a, c) f(z)]^{\prime}}{L(a, c) g(z)}\right\}>-\alpha
$$

Now, from Theorem 2.1, we know that $I_{\lambda}(g) \varepsilon R(a, c ; \beta)$. Let

$$
\frac{z\left[L(a, c) I_{\lambda}(f)\right]^{\prime}}{L(a, c) I_{\lambda}(g)}=(1+a) h(z)-\alpha,
$$

where

$$
h(z)=1+c_{1} z+c_{2} z^{2}+\ldots
$$

Note that

$$
z\left[L(a, c) I_{\lambda}(f)\right]^{\prime}=(\lambda+1) L(a, c) f(z)-\lambda L(a, c) I_{\lambda}(f)
$$

which readily yields

$$
z^{2}\left[L(a, c) I_{\lambda}(f)\right]^{\prime \prime}=(\lambda+1) z[L(a, c) f(z)]^{\prime}-(\lambda+1) z\left[L(a, c) I_{\lambda}(f)\right]
$$

Now, differentiating both sides of (2.2) logarithmically and using (2.3) and (2.4), we obtain

$$
\frac{(\lambda+1) z[L(a, c) f(z))]^{\prime}}{z\left[L(a, c) I_{\lambda}(f)\right]^{\prime}}-\frac{(\lambda+1) L(a, c) g(z)}{L(a, c) I_{\lambda}(g)}=\frac{(1+\alpha) z h^{\prime}(z)}{(1+d a) h(z)-\alpha}
$$


or, equivalently,

$$
\frac{(\lambda+1) L(a, c) g(z)}{z\left[L(a, c) l_{\lambda}(J)\right]^{\prime}}\left\{\frac{z[L(a, c) f(z)]^{\prime}}{L(a, c) g(z)}-\frac{z\left[L(a, c) l_{\lambda}(J)\right]^{\prime}}{L(a, c) l_{\lambda}(g)}\right\}=\frac{(1+\alpha) z h^{\prime}(z)}{(1+\alpha) h(z)-\alpha}
$$

After simplification, and taking

$$
\frac{z\left[L(a, c) I_{\lambda}(g)\right]^{\prime}}{L(a, c) I_{\lambda}(g)}=(1+\beta) H(z)-\beta,
$$

where $\operatorname{ReH}(z)=h_{1}>0$ and $\beta>-1$, we have, from (2.5),

$$
\frac{z[L(a, c) f(z)]^{\prime}}{L(a, c) g(z)}=(1+\alpha) h(z)-\alpha+\frac{(1+\alpha) z h^{\prime}(z)}{(1+\beta) H(z)-\beta+\lambda}
$$

or

$$
\frac{z[L(a, c) f(z)]^{\prime}}{L(a, c) g(z)}+\alpha=(1+\alpha) h(z)+\frac{(1+\alpha) z h^{\prime}(z)}{(1+\beta) H(z)-\beta+\lambda}
$$

We form the function $\phi(u, v)$ by taking

$$
u=h(z) \text { and } v=z h^{\prime}(z)
$$

in (2.6) as

$$
\phi(u, v)=(1+\alpha) u+\frac{(1+\alpha) v}{(1+\beta) H(z)-\beta+\lambda}
$$

It is clear that the function $\phi(u, v)$ defined by (2.7) satisfies conditions (i) and (ii) of Lemma 2.1 easily. To verify condition (iii), we proceed as follows.

$$
\operatorname{Re} \phi\left(i u_{2}, v_{1}\right)=\frac{(1+\alpha) v_{1}\left\{(1+\beta) h_{1}-\beta+\lambda\right\}}{\left[(1+\beta) h_{1}-\beta+\lambda\right]^{2}+\left[(1+\beta) h_{2}\right]^{2}}
$$

where $H(z)=h_{1}+i h_{2}, h_{1}$ and $h_{2}$ being the functions of $x$ and $y$ and $\operatorname{ReH}(z)=h_{1}>0$.

By putting $v_{1} \leq-\frac{1}{2}\left(1+u_{2}^{2}\right)$, we obtain

$$
\left.\operatorname{Re} \phi\left(i u_{2}, v_{1}\right) \leq-\frac{(1+d a)\left(1+u_{2}^{2}\right)\left\{(1+\beta) h_{1}-\beta+\lambda\right\}}{\left[(1+\beta) h_{1}-\beta+\lambda\right]^{2}+\left[(1+\beta) h_{2}\right]^{2}} \leq 0\right)
$$

Hence, by Lemma $2.1, \operatorname{Reh}(z)>0$ and this implies that $I_{\lambda}(f) \varepsilon T(a, c ; \alpha, \beta)$. This proves our theorem.

Corollary 2.1. Let $f \varepsilon T(a, c ; \alpha, \beta)$. Then, for $\lambda \geq \alpha, \beta>-1 L(a, c) I_{\lambda}(f) \varepsilon K$

Proof: From Theorem 2.2, we clearly see that $L(a, c) I_{\lambda}(f) \varepsilon K$. The second assertion follows easily from the fact that

$$
L(a, c) I_{\lambda}(f)=I_{\lambda}(L(a, c) f(z))
$$

Next we have:

Theorem 2.3. Let $f \varepsilon T^{*}(a, c ; \alpha, \beta)$. Then for $\lambda \geq \alpha, \beta>-1, I_{\lambda}(f)$ also belongs to $T^{*}(a, c ; \alpha, \beta)$.

Proof: Since

$$
f \varepsilon T^{*}(a, c ; \alpha, \beta) \leftrightarrow z f^{\prime} \varepsilon T(a, c ; \alpha, \beta),
$$

we observe, using Theorem 2.2, that

$$
I_{\lambda}\left(z f^{\prime}\right) \varepsilon T(a, c ; \alpha, \beta)
$$


and this implies that

$$
z\left(I_{,}(f)\right)^{\prime} \varepsilon T(a, c ;(x, \beta) .
$$

Hence $I_{l,}(f) \in T *(a, c ; u, \beta)$. This completes the proof.

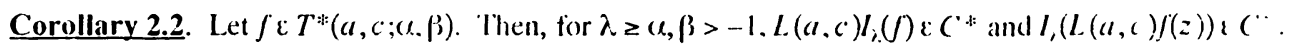

\section{REFERENCES}

1. Bernardi, S. D. "Convex and Starlike Univalent Functions," Trans, Amer. Matl. Soc., 1.35 (1969), 429-446.

2. Carlson, B. C. and Shaffer, D. B. "Starlike and Pre-starlike Hypergeometric Functions," SIAM J. Math. Anal., 15 (1984), 737-745.

3. Libera, R. J. "Some Radius of Convexity Problems," Duke Math. J. (1964), 143-158.

4. Libera, R. J. "Some Classes of Regular Univalent Functions," Proc. Amer. Math. Soc. 16 (1905), 755-758.

5. Livingston, A. E. "On the Radius of Univalence of Certain Analytic Functions," Proc. Amer. Math. Soc. 17 (1966), 352-357.

6. Miller, S. S. "Differential Inequalities and Caratheodory Functions," Bull. Amer. Math. Soc. $\underline{\text { sI }}$ (1975), 79-81.

7. Noor, K. I. "On Quasi-Convex Functions and Related Topics," Internat. J. Math. \& Math. Sci. 10 (1987), 241-258.

8. Robertson, M. S. "On the Theory of Univalent Functions," Ann. Math. 37 (1936), 241-258.

9. Srivastava, H. M. and Owa, S. "Some Characterization and Distortion Theorems Involving Fractional Calculus, Generalized Hypergeometric Functions, Linear Operators and Certain Subclasses of Analytic Functions," Nagoya Math. J. 106 (1987), 1-28. 


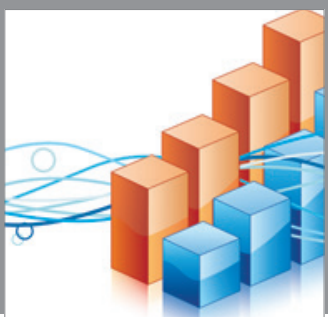

Advances in

Operations Research

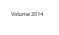

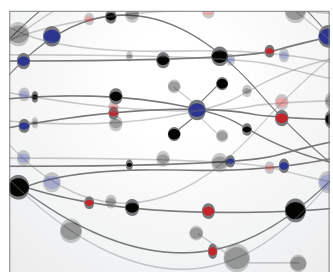

\section{The Scientific} World Journal
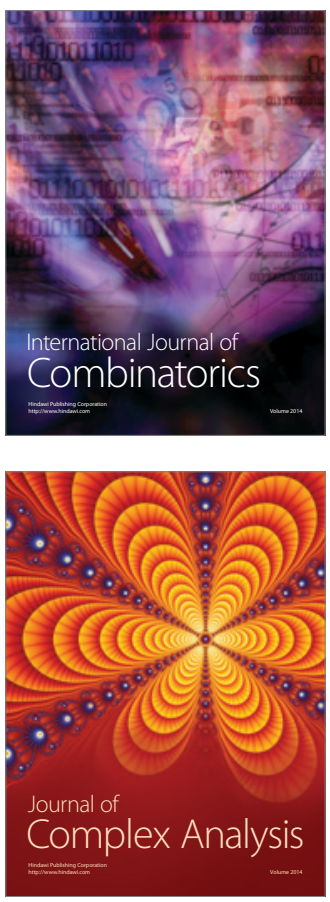

International Journal of

Mathematics and

Mathematical

Sciences
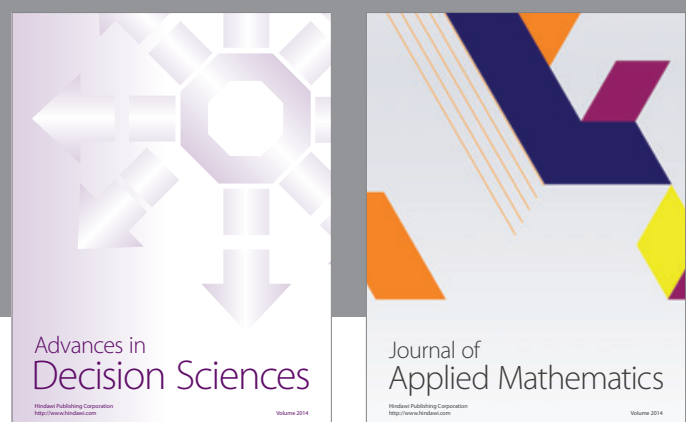

Journal of

Applied Mathematics
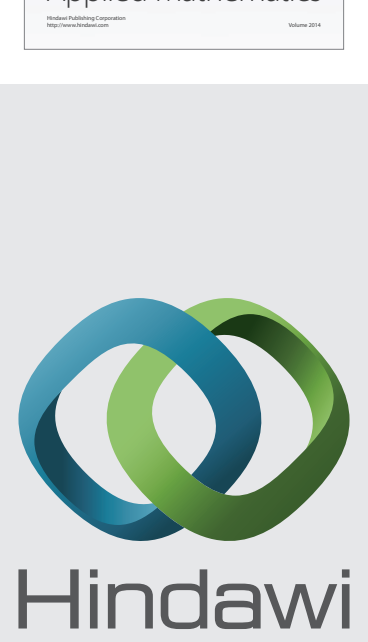

Submit your manuscripts at http://www.hindawi.com
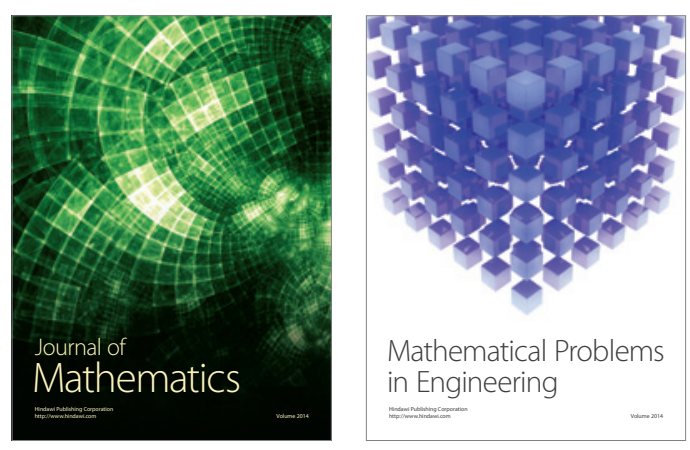

Mathematical Problems in Engineering
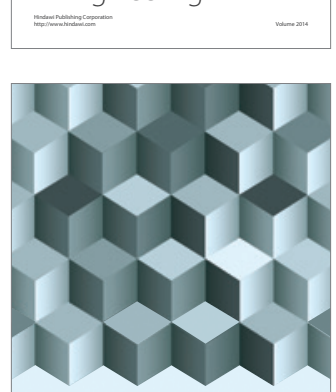

Journal of

Function Spaces
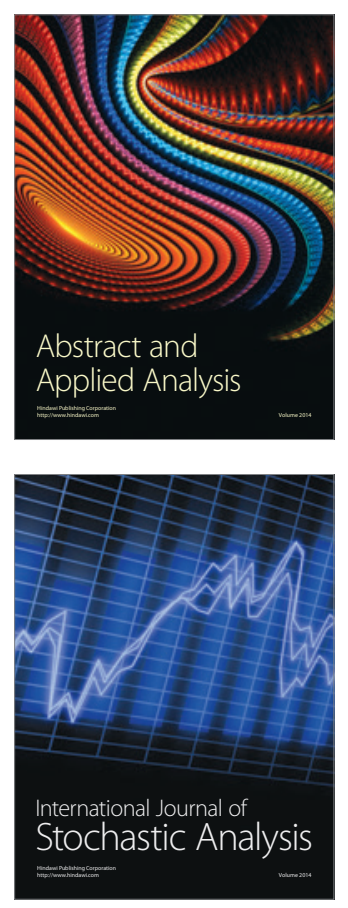

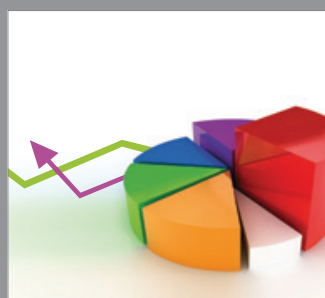

ournal of

Probability and Statistics

Promensencen
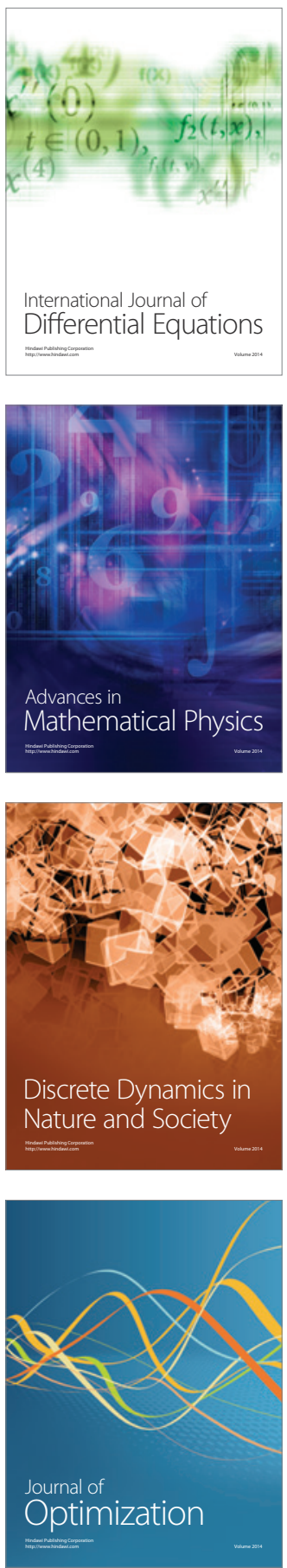